\title{
Towards the design of a prosthetic underactuated hand
}

\author{
T. Laliberté, M. Baril, F. Guay, and C. Gosselin \\ Département de Génie Mécanique, Université Laval, Québec, QC, G1V 0A6, Canada
}

Received: 25 February 2010 - Revised: 30 June 2010 - Accepted: 19 August 2010 - Published: 22 December 2010

\begin{abstract}
This paper presents recent advances in the design of an underactuated hand for applications in prosthetics. First, the design of the fingers is addressed. Based on previous experiments with prototypes developed in the past, new tendon routings are proposed that lead to a more effective transmission of the forces. A novel elastic tendon routing is also proposed for the passive opening of the hand. A simplified static analysis of the fingers is proposed to support the results. Then, a new kinematic design of the thumb is presented. The thumb is designed to perform out-of-the-plane motions in order to broaden the variety of possible grasps. A mechanism for the implementation of underactuation between the fingers is proposed that alleviates the friction problems encountered in earlier hand designs. Finally, a prototype of the hand is briefly described and typical grasps are shown.
\end{abstract}

This paper was presented at the IFToMM/ASME International Workshop on Underactuated Grasping (UG2010), 19 August 2010, Montréal, Canada.

\section{Introduction}

Underactuation will probably be an important feature in future prosthetic hands. Indeed, reducing the number of actuators without significantly compromising performance has many advantages such as reducing weight, cost and complexity of control.

Although research is pursued in order to allow users of prostheses to control many degrees of actuation (Alstrom et al., 1981; Chu et al., 2007; Castellini et al., 2008), the number of degrees of actuation is still limited. In this context, underactuation is a very attractive feature. Underactuation can be introduced in the fingers using link mechanisms (Shimojima et al., 1987; Laliberté and Gosselin, 1998; Dubey and Crowder, 2002). Although large forces can be obtained with this approach, the resulting fingers are significantly thicker than human fingers. In order to obtain more compact fingers, cable-driven mechanisms can be used (Hirose and Umetani, 1978; Crismal et al., 1996). Also, many efforts have been made in order to include underactuation between the fingers of a hand (Rakic, 1989; Fukaya et al., 2000; Birglen and Gosselin, 2006). Finally, the use of underaction in prosthetic devices is increasingly popular (Herder and Visser, 2000; Ky- berd et al., 2001; Massa et al., 2002; Dollar and Howe, 2007; Kamikawa and Maeno, 2008). For a review of underactuated hands, see Birglen et al. (2008).

In this paper, the ongoing design of a prosthetic underactuated hand is presented. The design is based on an anthropomorphic underactuated hand previously developed in the Robotics Laboratory at Laval University. The new design includes several improvements that mainly cover the underactuation within fingers, the passive opening of the fingers, the thumb implementation and the actuation transmission between the fingers.

\section{Previous work}

In Gosselin et al. (2008), an underactuated anthropomorphic robotic hand was presented that includes 15 degrees of freedom and a single actuator. The hand automatically adapts to the shape of objects and the fingers are designed to optimize grasping criteria, mainly the resulting force on the object and force distribution among the phalanges. At rest, the hand is completely open and when it is operated, the four fingers, then the thumb, close together until they make contact with an object. 


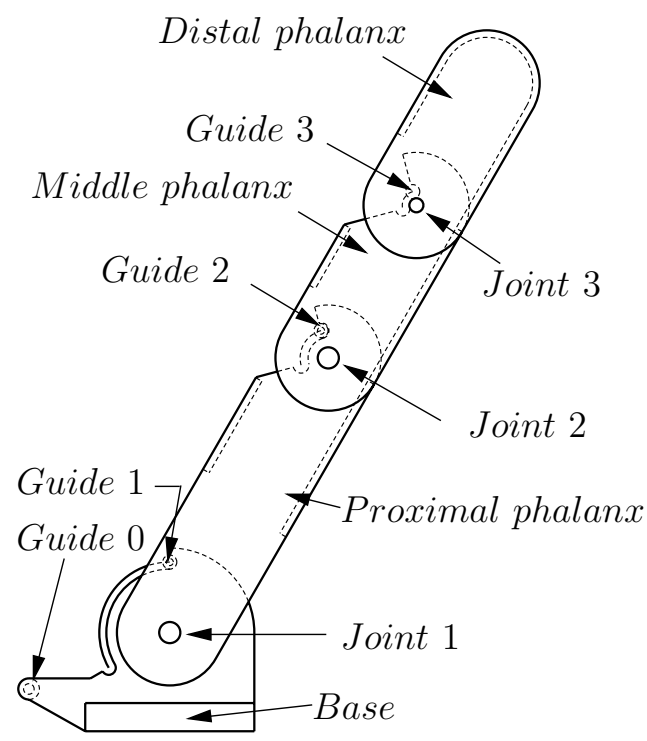

Figure 1. Design of the fingers proposed in Gosselin et al. (2008), which includes guiding rods.

Like the tendons of a human hand, finger actuation (power transmission) is performed through cables. The torque ratio between the joints is mainly dictated by the position of guiding points on each phalanx. In the prototype presented in Gosselin et al. (2008), the fingers are normally maintained open by torsional springs inserted at each of the joints. The order of closure of each phalanx is dictated by the actuation torque available at each joint and the opening torque from the torsional springs. The geometric design of the fingers is presented in Fig. 1 (see Gosselin et al., 2008, for more details).

It is worth mentioning that two different mechanisms have been studied using the prototype presented in Gosselin et al. (2008) to achieve underactuation and coupling between the fingers. In the first mechanism - which did not work very well mainly because of friction - the thumb and the fingers are all linked by an underactuated mechanism. In the second mechanism - which worked better - the thumb is coupled to the other fingers, which are underactuated. These mechanisms are composed of cables, pulleys, guides and slides, and are described in Gosselin et al. (2008).

The thumb is placed such that it is in opposition with the index finger and middle finger and such that it is oriented to point slightly towards the centre of the hand. A plastic prototype of the hand was built using rapid prototyping. The joint pins were made of metal. Kite cables were used for the tendons, which provided high stiffness, flexibility and relatively low friction. In order to provide manual actuation of the hand, the prototype was equipped with a handle, which is convenient for the testing and demonstration of the hand in a general context. The prototype proposed in Gosselin et al. (2008) is shown in Fig. 2.

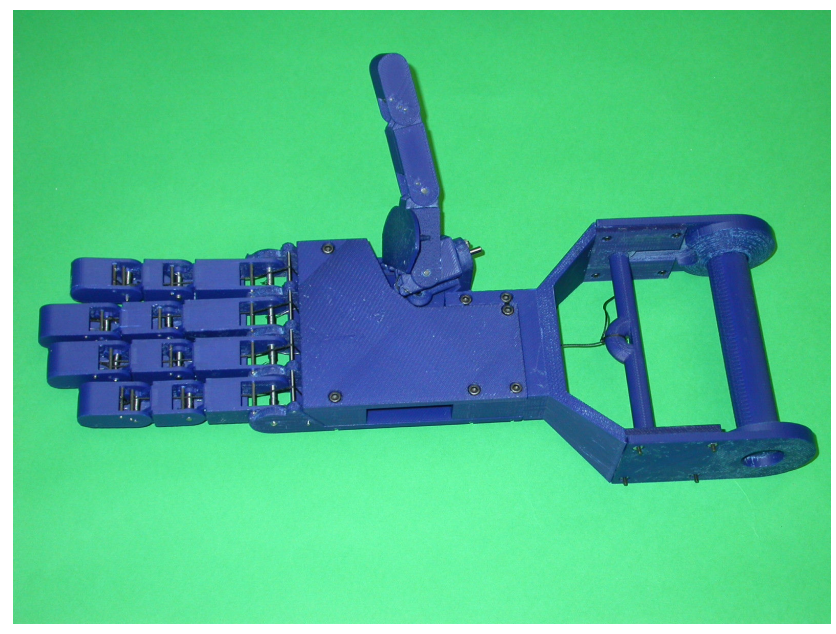

Figure 2. Prototype of underactuated hand proposed in Gosselin et al. (2008).

Experiments demonstrated that the hand is performing well for enveloping grasps, which involve contact with all the phalanges. The grasps are generally firm and stable and have a human-like appearance.

However, the experiments also revealed several weaknesses. As opposed to what was observed for enveloping grasps, the hand often performed poorly whith pinch grasps of small objects, which involve contact with only the tip of the fingers. This is due to a lack of synchronization of the closing motion of the thumb and fingers as well as to the slanted orientation of the thumb, which is not ideal for pinch grasps. Friction is also an important issue. For instance, the efficiency of the transmission system was estimated to only $35 \%$. This is mainly explained by the use of small rods as guiding points that add friction as the cables rub directly on them. This is also explained by the sliding joints located in the palm. The sliders can even jam the mechanism which makes it difficult to predict the trajectory of the fingers. With the use of guiding pins, the torques and forces in the fingers are not constant during the closing of the fingers since the lever arms are not constant. One of the consequences is that the force needed to start the motion of the fingers is too large. Finally, the use of torsional springs to open the fingers makes the assembly of the fingers quite tedious.

Consequently, a new prototype has been developed to address the above issues. Changes have been made to the fingers, thumb and underactuated mechanisms. Also, the new hand is attached directly to a lower arm prosthesis to verify experimentally the advantages and disadvantages of the hand as a prosthesis. 


\section{Improved finger design}

Several improvements have been made to the fingers of the new prototype. Firstly, the guiding pins of the previous version have been replaced by pulleys. This reduces the friction and provides constant ratios at each joint. This modification adds significant flexibility to the design, since the ratios can be easily controlled by changing the diameter of the pulleys. Optimal combinations are obtained from numerical simulations. Another important change is that the opening of the fingers is now produced by an opening cable rather than torsional springs. The force in the opening cable can be obtained from a spring attached in series with the cable. However, it is more compact to replace the cable and spring by a bungee cable, which integrates the cable and spring in the same component. Similarly to the closing mechanism, the ratios of the pulleys in the routing of the opening cable can be easily controlled by changing the diameter of the pulleys. Therefore, there are now two independent tendons per finger, an active tendon for closing and a passive tendon for opening. This significantly simplifies assembly and provides better compactness. This new type of finger is consequently simpler, more efficient and more accurate.

As mentioned above, the fingers are composed of two independent tendons directed through small pulleys. For the actuation tendon, two slightly different routing options have been developed.

\subsection{Actuation routing with idler on the middle phalanx}

For the actuation tendon, the topology of the routing is similar to that presented in Crismal et al. (1996). However, in order to obtain the desired range of motion $-0^{\circ}$ to $90^{\circ}$ at each joint - the pulleys are located differently. The tendon is attached to the inside of the distal phalanx, routed down the finger and fixed on the mechanism providing the underactuation between the fingers, located in the palm of the hand. As shown in Fig. 3, the cable is maintained in contact with the pulleys by idlers for any configuration of the finger. This provides a constant torque at all joints for all possible configurations. The distal phalanx does not require sheave since the cable does not move in it. Indeed, the closure of this phalanx simply affects the winding on the distal pulley and this movement generates very little friction.

\subsection{Actuation routing without idler on the middle phalanx}

In the second routing, illustrated in Fig. 4, an idler is added to the base of the finger in order to displace the input tendon in the plane of the underactuation mechanism located in the palm. Because of the idler in the base, the idler in the proximal phalanx can be displaced close to the middle joint. This allows to keep the cable in contact with the pulleys in all configurations without the use of an idler in the middle phalanx. The removal of the middle idler minimizes fric-

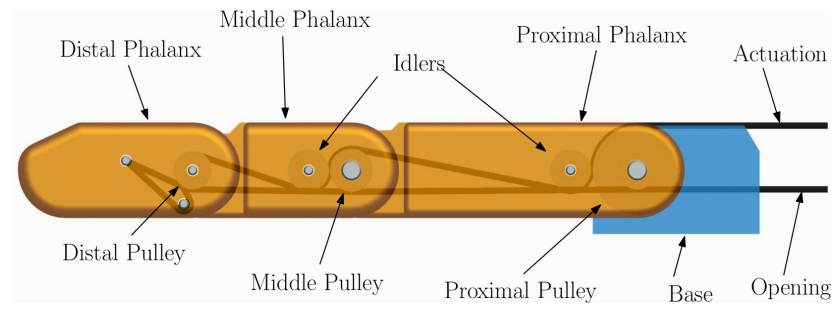

Figure 3. CAD model of the proposed finger with the actuation routing using an idler on the middle phalanx.

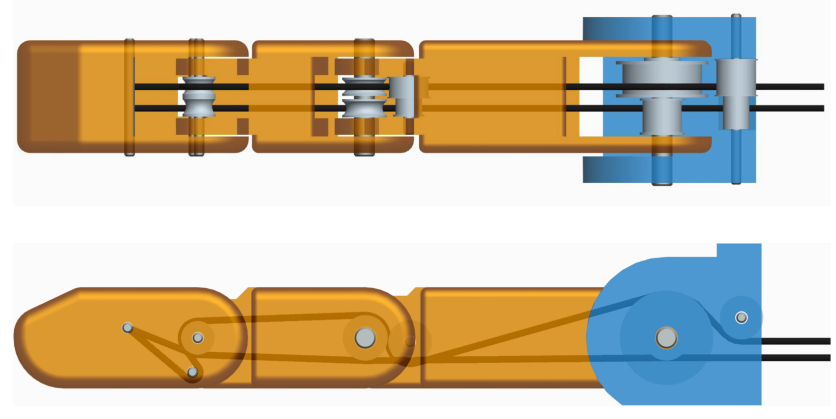

Figure 4. CAD model of the proposed finger with the actuation routing that does not require an idler on the middle phalanx.

tion. This routing has been implemeted in the prototype. It is noted that as long as the cable is maintained in contact with the pulleys, the two mechanisms are kinematically equivalent.

\subsection{Routing of the opening cable}

As illustrated in Figs. 3 and 4, the tendon used to open the finger is also attached to the distal phalanx and routed to the palm where it is fixed. Compared to the actuation tendon, it passes on the opposite side of the joints of each phalanx to produce the opening of the finger. This tendon is also always in contact with the pulleys in the joints without the need of idlers, which minimizes friction. Similarly to the actuation torques, the opening torques are independent from the configuration of the finger and only depend on the tension in the bungee cable.

\subsection{Static modelling of the fingers}

The static model of the fingers was developed using the procedure given in Birglen et al. (2008). According to the latter reference, the force transmission between the actuator - here the force on the tendon at the base of the finger - and the contact forces on the phalanges can be written as:

$\boldsymbol{f}=\mathbf{J}^{-T} \mathbf{T}^{-T} \boldsymbol{t}$

where $\boldsymbol{f}=\left[f_{1}, f_{2}, f_{3}\right]^{T}$ is the vector of phalanx contact forces with the object, matrix $\mathbf{J}$ is a lower triangular matrix 
characteristic of the contact locations - and friction, if modelled - referred to as the Jacobian matrix of the finger, matrix $\mathbf{T}$ is the transmission matrix, characterizing the transmission used and its geometry, and vector $\boldsymbol{t}$ is the vector of actuating torques at the joints. The analytical expression of the matrices defined above can be found in Birglen et al. (2008) for linkage-driven fingers and tendon-driven fingers.

\subsubsection{Closing sequence}

A knowledge of the torques acting internally at each joint of a finger was needed in order to determine the closing sequence of the finger. The model developed provides the magnitude of the response torque vector, $\tau$, based on an actuation torque vector, $\tau_{\mathrm{a}}$, an opening torque vector, $\boldsymbol{\tau}_{\mathrm{r}}$, and a torque vector due to gravity, $\tau_{\mathrm{g}}$. Mathematically, Eq. (1) can be reformulated as

$f=\mathbf{J}^{-T} \boldsymbol{\tau}$

with

$\tau=\tau_{\mathrm{a}}+\tau_{\mathrm{r}}+\tau_{\mathrm{g}}$

Then, the actuation torque vector is written as

$\boldsymbol{\tau}_{\mathrm{a}}=\mathbf{T}_{\mathrm{a}}^{-T} \boldsymbol{t}_{\mathrm{a}}$

where $\mathbf{T}_{\mathrm{a}}$ is the transmission matrix which relates the actuation joint velocity to the time derivatives of the joint coordinates. For this design, one has

$\mathbf{T}_{\mathrm{a}}=\left[\begin{array}{ccc}1 & \frac{-r_{3 \mathrm{a}}}{r_{1 \mathrm{a}}} & \frac{-r_{5 \mathrm{a}}}{r_{1 \mathrm{a}}} \\ 0 & 1 & 0 \\ 0 & 0 & 1\end{array}\right]$

where $r_{1 \mathrm{a}}, r_{3 \mathrm{a}}$ and $r_{5 \mathrm{a}}$ are the radii of the proximal, middle and distal actuation pulleys respectively and $\boldsymbol{t}_{\mathrm{a}}$ is the input torque vector defined as $\boldsymbol{t}_{\mathrm{a}}=\left[T_{\mathrm{a}}, 0,0\right]^{T}$. Similarly, the opening torque vector is written as

$\tau_{\mathrm{r}}=\mathbf{T}_{\mathrm{r}}^{-T} \boldsymbol{t}_{\mathrm{r}}$

where $\mathbf{T}_{\mathrm{r}}$ is the transmission matrix which, in this design, is exactly the same matrix as for the actuation apart from the pulley radii which are different for the opening routing, namely

$\mathbf{T}_{\mathrm{r}}=\left[\begin{array}{ccc}1 & \frac{-r_{3 \mathrm{r}}}{r_{1 \mathrm{r}}} & \frac{-r_{5 \mathrm{r}}}{r_{1 \mathrm{r}}} \\ 0 & 1 & 0 \\ 0 & 0 & 1\end{array}\right]$

where $r_{1 \mathrm{r}}, r_{3 \mathrm{r}}$ and $r_{5 \mathrm{r}}$ are the radii of the proximal, middle and distal opening pulleys respectively and $t_{\mathrm{r}}$ is the opening torque vector defined as $\boldsymbol{t}_{\mathrm{r}}=\left[T_{\mathrm{r}}, 0,0\right]^{T}$.

Finally, the torque vector due to gravity is written as $\tau_{\mathrm{g}}=$ $\left[\tau_{\mathrm{g} 1}, \tau_{\mathrm{g} 2}, \tau_{\mathrm{g} 3}\right]^{T}$ where the three entries describe the effect of

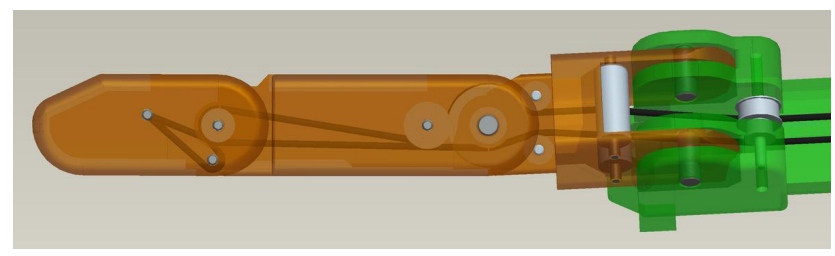

Figure 5. CAD model of the thumb.

gravity on each joint, namely:

$$
\begin{aligned}
\tau_{\mathrm{g} 1} & =\cos (\varphi)\left(\left[m_{1} g c_{\mathrm{g} 1} \cos \left(\gamma_{0}+\gamma_{1}\right)\right]\right. \\
& +\left[m_{2} g\left(c_{\mathrm{g} 2} \cos \left(\gamma_{0}+\gamma_{1}+\gamma_{2}\right)+l_{1} \cos \left(\gamma_{0}+\gamma_{1}\right)\right)\right] \\
& +\left[m _ { 3 } g \left(c_{\mathrm{g} 3} \cos \left(\gamma_{0}+\gamma_{1}+\gamma_{2}+\gamma_{3}\right)+l_{2} \cos \left(\gamma_{0}+\gamma_{1}+\gamma_{2}\right)\right.\right. \\
& \left.\left.\left.+l_{1} \cos \left(\gamma_{0}+\gamma_{1}\right)\right)\right]\right) \\
\tau_{\mathrm{g} 2} & =\cos (\varphi)\left(\left[m_{2} g c_{\mathrm{g} 2} \cos \left(\gamma_{0}+\gamma_{1}+\gamma_{2}\right)\right]\right. \\
& +\left[m _ { 3 } g \left(c_{\mathrm{g} 3} \cos \left(\gamma_{0}+\gamma_{1}+\gamma_{2}+\gamma_{3}\right)\right.\right. \\
& \left.\left.\left.+l_{2} \cos \left(\gamma_{0}+\gamma_{1}+\gamma_{2}\right)\right)\right]\right) \\
\tau_{\mathrm{g} 3} & =\cos (\varphi)\left(m_{3} g c_{\mathrm{g} 3} \cos \left(\gamma_{0}+\gamma_{1}+\gamma_{2}+\gamma_{3}\right)\right)
\end{aligned}
$$

where $g$ is the gravitational acceleration, $m_{i}$ is the mass of the $i$-th phalanx, $c_{g i}$ is the distance from the $i$-th joint to the centre of mass of the $i$-th phalanx and $l_{i}$ is the length of the $i$-th phalanx. Furthermore, $\varphi$ and $\gamma_{0}$ are the first two angles of the Tait-Bryan's convention which is used here to describe the orientation of the hand (pitch and roll). Finally, angle $\gamma_{i}$ is the angle describing the rotation of the $i$-th joint of the finger.

\section{Improvement of the thumb design}

Several changes were also made to the thumb in the new prototype. Like the new fingers, the thumb is now composed of two independent sets of cables and pulleys, one for closing and another one for the opening. However, a twist rotation is introduced between the proximal phalanx and the middle joint in order to explore the possibilites of motion out of a single plane and to broaden the variety of possible grasps (see Fig. 5). More precisely, the proximal joint of the thumb is perpendicular to the axes of the fingers, allowing a lateral motion of the thumb. The middle joint is rotated 25 degrees from the axes of the fingers (65 degrees from the proximal axis of the thumb) and points towards the centre of the hand. This rotation allows a more anthropomorphic behaviour and experiments show that it improves the quality of the grasps. In order to cope with the rotation between the proximal and middle joints, four idlers are included to manage the routing of cables. The middle and distal phalanges are similar to those of the other fingers, but they include the first actuation routing and the actuation pulley of the middle joint is slightly larger in order to obtain more force on the thumb. The thumb is illustrated in Fig. 5. 


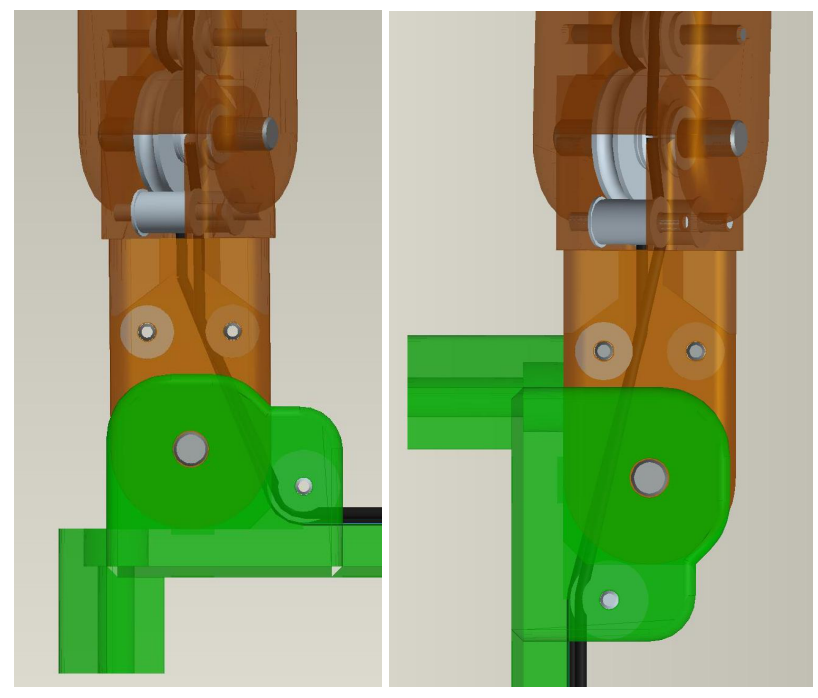

Figure 6. CAD model of the routing of the tendons of the thumb around the proximal joint: opposing configuration (left) and lateral configuration (right).

In this specific implementation, it is desired to obtain two possible configurations of the thumb: one opposing the other fingers and one located lateraly with respect to the other fingers. It is expected that this new feature will allow to pick up some objects on flat surfaces more easily while still allowing the performance of enveloping grasps. The configuration with the thumb facing the other fingers is mainly used for enveloping grasps. It can also be used for pinch grasps with the tip of the fingers, but with limited stability. The configuration with the thumb located lateraly allows to perform pinch grasps using the side of the closed index. In this configuration, the large surface of the side of the closed index allows more stable pinch grasps.

In order to select which configuration to use, a first option would be to include an additional actuator, which significantly complexifies the hand considering that all that is needed is a simple switch between two configurations. The suggested option is to ensure that the thumb freely stays in one of the two configurations and to select the other configuration by pushing laterally on the thumb with the other hand or against a fixed object. In order to obtain this feature, the actuation cables (closing and opening) can pass through the first axis when the thumb is moved laterally (see Fig. 6). Then, for each configuration, the passive opening cable tends to keep the thumb in place. It is noted that the active closing cable is slightly loose when not actuated, which eases the change of configuration. Then, when the thumb is closed, the closing cable further helps to keep the thumb in place.

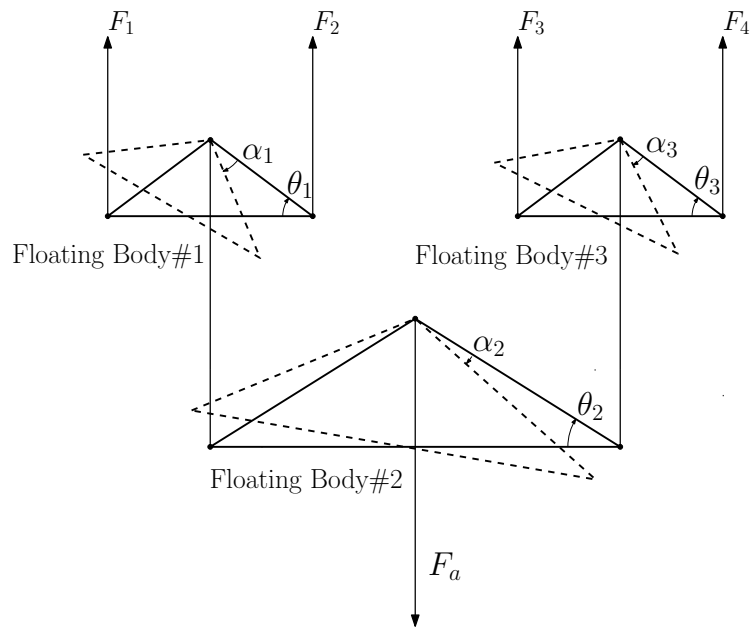

Figure 7. Schematic illustration of the mechanism allowing underactuation between the fingers.

\section{Mechanism for the underactuation between the fingers}

\subsection{Description of the mechanism}

The prototype presented here can be useful to test various mechanisms of transmission between the fingers. Indeed, a relatively large space is devoted to include different transmission mechanisms in the palm. Some mechanisms providing underactuation between the fingers have been developed (Baril et al., 2010). One of the most promising mechanisms is implemented and discussed here. As shown in Fig. 7, this mechanism is composed of three small seesaws connected together with cables. The geometric arrangement generates an equal force in each finger. When the hand is activated but does not make contact with an object, it simply closes and the entire mechanism moves downward, without any rotation of the rods. However, if there is a restriction on a finger, the smaller floating bodies will rotate to allow other fingers to continue their trajectory. The same phenomenon occurs if two consecutive fingers are subject to a restriction, except that the rotation will take place at the larger floating body. It is noted that the range of underactuation possible with this mechanism depends on the distance between the outputs. Indeed, the maximum relative motion of two fingers linked by a seesaw is given by the length of the seesaw. As a result, the closing motion of a finger can be slightly limited if its neighbour is kept fully open. A major distinction with the mechanisms developed in Gosselin et al. (2008) is that the mechanisms presented here are fully floating, i.e., they are not guided by sliders. This has the effect of decreasing friction and increasing precision. Also, the mechanism is much simpler since it does not need to be set anywhere in the palm. Finally, the thumb is coupled with the four fingers by attaching its tendon directly to the actuation tendon. As discussed 


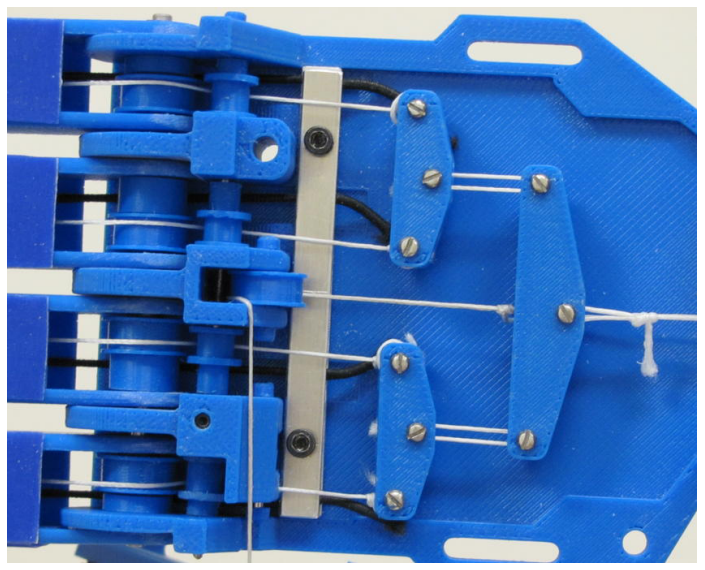

Figure 8. Mechanism allowing underactuation between the fingers and coupling with the thumb. The thumb has been removed for clarity. The thumb tendon is attached at the centre of the large seesaw.

in Gosselin et al. (2008) this configuration gives good practical results, mainly because the thumb works in opposition with the other fingers. The mechanism implemented is illustrated in Fig. 8.

\subsection{Static modelling of the underactuation between the fingers}

In Fig. 7, it should be noted that if the angle $\theta_{i}$ describing the geometry of a floating body is negative, then the triangle will be pointing towards the bottom instead of the top. This affects the kinematics of the mechanism for the cases of underactuation between the different outputs, as described in the following equations. To simplify the analysis, lateral movements between the triangles are neglected, i.e., it is assumed that the cables remain parallel. This assumption is valid as long as the lateral displacements are significantly smaller than the length of the cables. From the static equilibrium, one has

$F_{1}=\frac{c_{\mathrm{p} 1} c_{\mathrm{p} 2} F_{\mathrm{a}}}{c_{\mathrm{p} 1} c_{\mathrm{p} 2}+c_{\mathrm{p} 1} c_{\mathrm{m} 2}+c_{\mathrm{m} 1} c_{\mathrm{p} 2}+c_{\mathrm{m} 1} c_{\mathrm{m} 2}}$

$F_{2}=\frac{c_{\mathrm{m} 1} c_{p 2} F_{\mathrm{a}}}{c_{\mathrm{p} 1} c_{\mathrm{p} 2}+c_{\mathrm{p} 1} c_{\mathrm{m} 2}+c_{\mathrm{m} 1} c_{\mathrm{p} 2}+c_{\mathrm{m} 1} c_{\mathrm{m} 2}}$

$F_{3}=\frac{c_{\mathrm{p} 3} c_{\mathrm{m} 2} F_{\mathrm{a}}}{c_{\mathrm{p} 3} c_{\mathrm{p} 2}+c_{\mathrm{p} 3} c_{\mathrm{m} 2}+c_{\mathrm{m} 3} c_{\mathrm{p} 2}+c_{\mathrm{m} 3} c_{\mathrm{m} 2}}$

$F_{4}=\frac{c_{\mathrm{m} 3} c_{\mathrm{m} 2} F_{\mathrm{a}}}{c_{\mathrm{p} 3} c_{\mathrm{p} 2}+c_{\mathrm{p} 3} c_{\mathrm{m} 2}+c_{\mathrm{m} 3} c_{\mathrm{p} 2}+c_{\mathrm{m} 3} c_{\mathrm{m} 2}}$

where

$c_{\mathrm{p} i}=\cos \left(\alpha_{i}+\theta_{i}\right) \quad i=1,2,3$

$c_{\mathrm{m} i}=\cos \left(\alpha_{i}-\theta_{i}\right) \quad i=1,2,3$

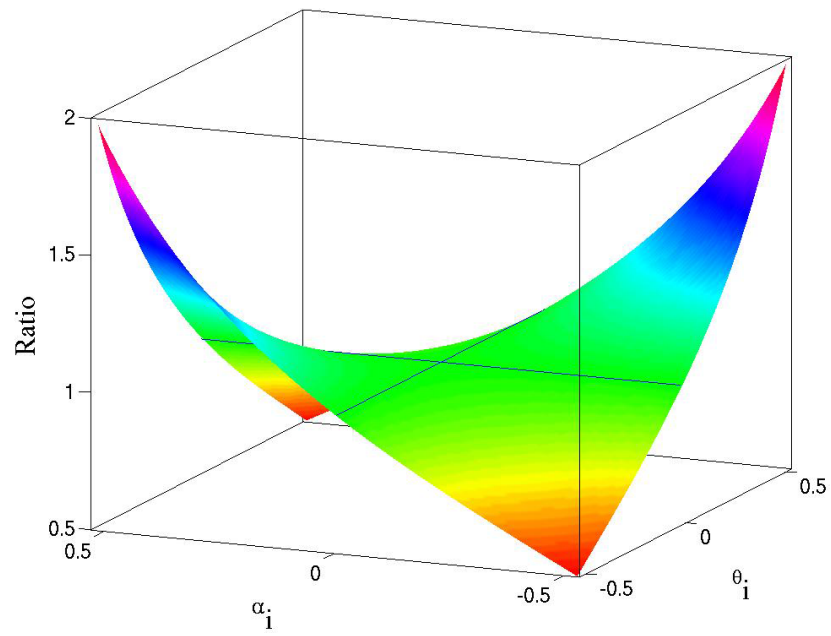

Figure 9. Ratio of the output forces of a triangular seesaw as a function of the floating body geometry $\left(\theta_{i}\right)$ and orientation $\left(\alpha_{i}\right)$.

When the triangular seesaw rotates, its two outputs will not be equal because the lever arms are changing. Figure 9 summarizes the effect of the angles $\theta_{i}$ and $\alpha_{i}$ on the ratio of the two output forces of a triangular seesaw. As a remark, if $\theta_{i}=0$ or if $\alpha_{i}=0$, then the two output forces are equal.

It is important to mention that it is preferable to let the triangles point downward $\left(\theta_{i}<0\right)$, since the mechanism is more likely to stay in an equilibrium configuration if a resistive force (for example friction) is slightly larger at one of the outputs. Then, an external perturbation will only slightly desynchronize the output positions. This is due to the fact that the seesaw applies more force on the output offering more resistance. On the contrary, if the triangles point upward $\left(\theta_{i}>0\right)$, the seesaw will flip on its side as soon as the resisting force is slight larger at one of the outputs. Then, a small external perturbation will strongly desynchronize the output positions. This is due to the fact that the seesaw applies a smaller force on the output with more resisting force. In practice, a small negative $\theta_{i}$ is suggested. This generally allows to maintain the synchronization of the mechanism when small perturbations, such as friction, are applied, but leads to relatively equal forces on four outputs locked at different positions.

\section{Hand integration}

The prototype of prosthetic hand is illustrated in Figs. 10, 11, 12,13 and 14. It is made of plastic and built using rapid prototyping. The connection between the hand and the prosthesis forearm is through a steel shaft with threads at both ends. This shaft is inserted tightly in a part made of rapid prototyping and the tip pointing inside the hand is fixed with a nut. For the other end, a small adapter is screwed on so the hand is compatible with the prosthesis forearm. For the actuation of the hand, the main cable actuating the mechanism in the 


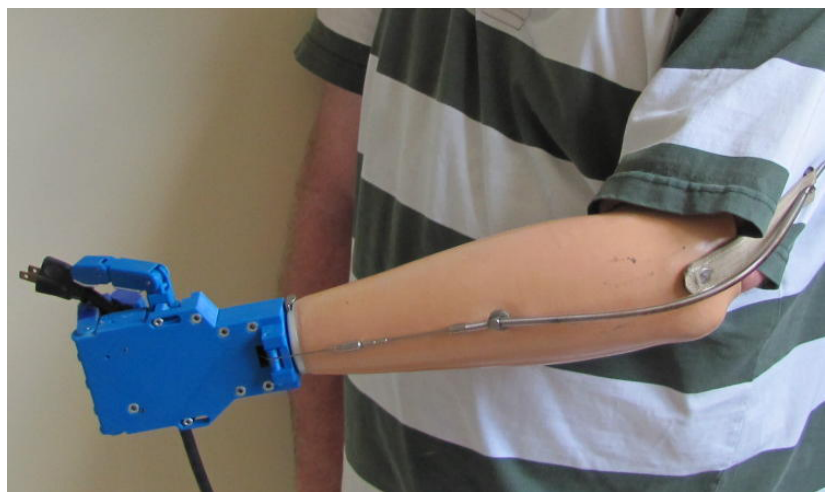

Figure 10. The prosthetic hand and forearm used by an amputee, grasping a plug.

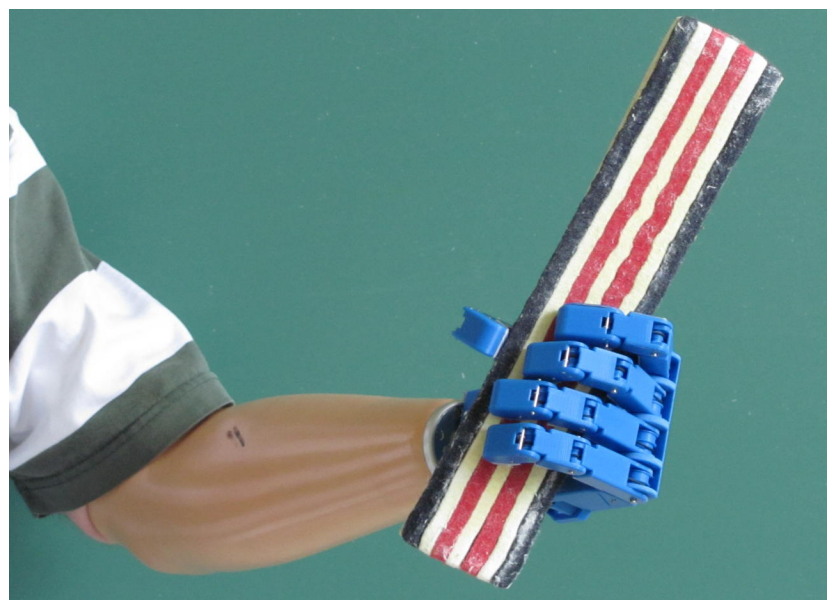

Figure 11. The prosthetic hand and forearm used by an amputee, grasping an eraser.

palm is simply attached to the steel cable of the prosthesis. The hand connected to the prosthesis forearm is illustrated in Figs. 10 and 11. The prosthesis is actuated through a harness by a motion of the shoulders of the amputee. It is noted that a handle can be attached to the hand in order to actuate it manually. Many objects have been grasped with enveloping grasps (ball, wine glass, bottle, etc.), pinch grasps (nut, small box, etc.) and lateral pinch grasps (spring, fork, paper, coin, etc.). As expected, the lateral pinch grasp is more stable than the opposing pinch grasp. Examples are illustrated in Figs. 12, 13 and 14.

In order to estimate the improvement of the efficiency of the prosthetic hand over the previous design, preliminary measurements of the forces on a grasped object have been performed with a setup similar to the one used in Gosselin et al. (2008). For instance, the actuation is applied by suspending weights (the forearm and harness are removed) and the force on the object is measured using a dynamometer.

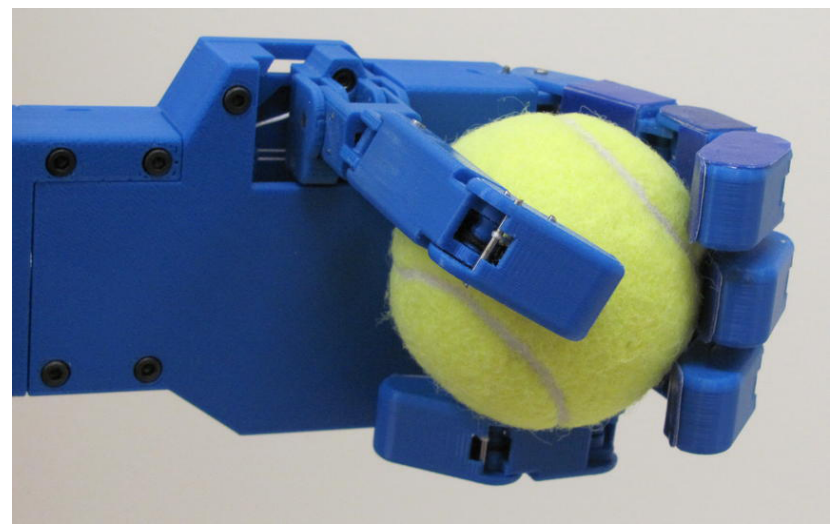

Figure 12. The prosthetic hand grasping a tennis ball with an enveloping grasp.

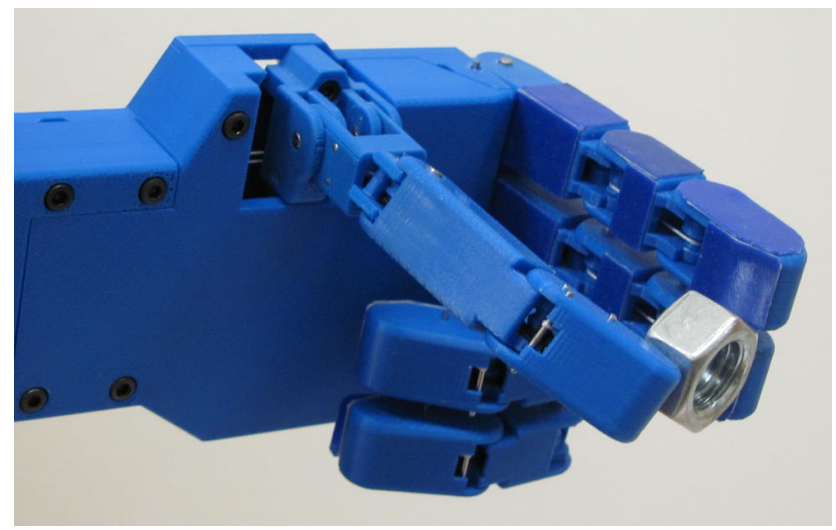

Figure 13. The prosthetic hand grasping a nut with an opposing pinch grasp.

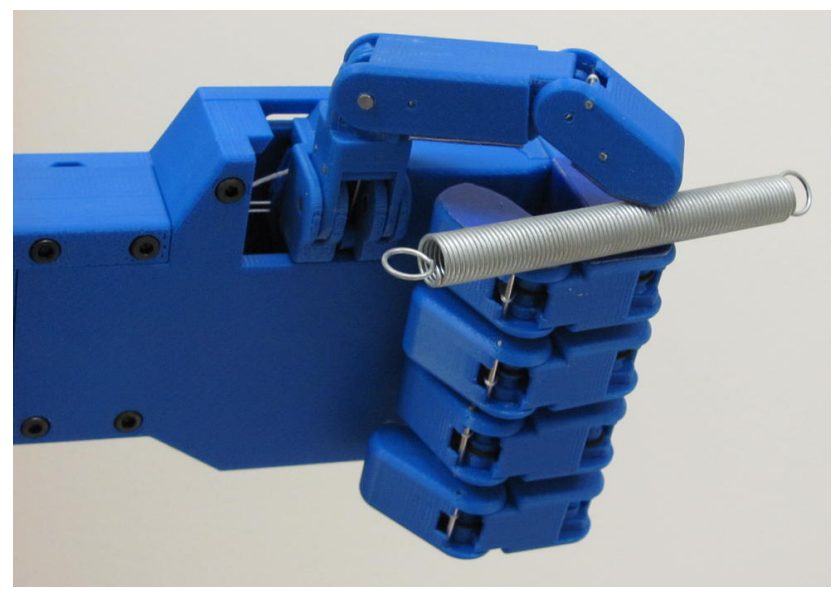

Figure 14. The prosthetic hand grasping a spring with a lateral pinch grasp. 
Two situations are studied. First, the fingers are closed towards the object, which reproduces the conditions corresponding to the grasping of an object. Second, the object is pushed towards the fingers, which corresponds to an external force applied on a grasped object. The efficiency can then be estimated from the ratio between the forces measured. The estimated efficiency of the prosthetic hand is 0.70 , which is a significant improvement over the efficiency of 0.35 of the previous prototype. This improved efficiency increases the capability to apply grasping forces.

\section{Conclusions}

In this paper, the ongoing design of a prosthetic hand is presented. The prosthetic hand is based on an anthropomorphic robotic hand previously developed. The actuation mechanism of the fingers is improved in order to reduce friction and obtain a constant transmission force. Also, the prototype is designed to test different mechanisms of underactuation between the fingers. A promising version, based on seesaw mechanisms, is included. It leads to reduced friction and improved behaviour. Finally a new thumb, which allows motion outside of a single plane is introduced. This thumb allows enveloping and pinch grasps with other fingers and lateral pinch grasps between the thumb and the side of the index finger. Preliminary experiments conducted with the prototype demonstrate the effectiveness of each type of grasp. Although preliminary force measurements confirmed a signifiant reduction of the friction in the hand, additional force measurements will be performed in order to better characterize the prosthetic hand. Also, the qualitative performance of the grasp on a variety of objects will be evaluated. Finally, the work presented here is mainly devoted to the underactuated transmission mechanisms. Therefore, significant work is still required in order to address other issues pertaining to prosthetics, such as easthetics, actuation means, covering, and others.

Acknowledgement. The authors gratefully acknowledge the financial support of the Natural Sciences and Engineering Research Council of Canada (NSERC) as well as the Canada Research Chair program.

Edited by: J. L. Herder

Reviewed by: two anonymous referees

\section{References}

Almström, C., Herberts, P., and Korner, L.: Experience with Swedish Multifunctional Prosthetic Hands Controlled by Pattern Recognition of Multiple Myoelectric Signals, Int. Orthop., 5, 1521, 1981.

Baril, M., Laliberté, T., Guay, F., and Gosselin, C.: Static Analysis of Single-Input/Multiple-Output Tendon-Driven Underactuated Mechanisms for Robotic Hands, in: Proceedings of the ASME IDETC/CIE 2010, Montreal, Canada, 2010.
Birglen, L. and Gosselin, C.: Force Analysis of Connected Differential Mechanisms: Application to Grasping, Int. J. Rob. Res., 25, 1033-1046, 2006.

Birglen, L., Laliberté, T., and Gosselin, C.M.: Underactuated Robotic Hands, Springer Tracts in Advanced Robotics, 40, 244 pp., 2008.

Castellini, C., van der Smagt, P., Sandini, G., and Hirzinger, G.: Surface EMG for Force Control of Mechanical Hands, in: Proceedings of the 2008 IEEE International Conference on Robotics and Automation, Pasadena, CA, 2008.

Chu, J. U., Moon, I., Lee, Y. J., Kim, S. K., and Mun, M. S.: A Supervised Feature-Projection-Based Real-Time EMG Pattern Recognition for Multifunction Myoelectric Hand Control, IEEE/ASME Trans. Mechatron., 12, 282-290, 2007.

Crisman, J. D., Kanojia, C., and Zeid, I.: Graspar: A Flexible, Easily Controllable Robotic Hand, IEEE Rob. Autom. Mag., June, 32-38, 1996.

Dollar, A. M. and Howe, R. D.: The SDM Hand as a Prosthetic Terminal Device: A Feasibility Study, in: Proceedings of the 2007 IEEE International Conference on Rehabilitation Robotics (ICORR), Noordwijk, Netherlands, 2007.

Dubey, V. N. and Crowder, R. M.: A Finger Mechanism for Adaptive End Effectors, in: Proceedings of 2002 ASME Design Engineering Technical Conferences, Montreal, Canada, 2002.

Fukaya, N., Toyama, S., Asfour, T., and Dillmann, R.: Design of the Tuat/Karlsruhe Humanoid Hand, in: Proceedings of 2000 IEEE/RSJ International Conference on Advanced Robotics, 3, Takamatsu, Japan, 1754-1759, 2000.

Gosselin, C., Pelletier, F., and Laliberté, T.: An Anthropomorphic Underactuated Robotic Hand with 15 Dofs and a Single Actuator, in: Proceedings of the 2008 IEEE International Conference on Robotics and Automation, Pasadena, CA, 2008.

Herder J. L., Visser H. de: Force Directed Design of a Voluntary Closing Hand Prosthesis, in: Proceedings ASME DETC 26th Biennial Mechanisms and Robotics Conference, Baltimore, MD, 2000.

Hirose, S. and Umetani, Y.: The Development of Soft Gripper for the Versatile Robot Hand, Mech. Mach. Theory, 13, 351-358, 1978.

Kamikawa, Y. and Maeno, T.: Underactuated Five-Finger Prosthetic Hand Inspired by Grasping Force Distribution of Humans, in: Proceedings of the IEEE/RSJ International Conference on Intelligent Robots and Systems, Nice, France, 2008.

Kyberd, P. J., Light, C., Chappell, P. H., Nightingale, J. M., Whatley, D., and Evans, M.: The Design of Anthropomorphic Prosthetic Hands: A Study of the Southampton Hand, Robotica, 19, 593-600, 2001.

Laliberté, T. and Gosselin, C.: Simulation and Design of Underactuated Mechanical Hands, Mech. Mach. Theory, 33, 39-57, 1998.

Massa, B., Roccella, S., Carrozza, M. C., and Dario, P.: Design and Development of an Underactuated Prosthetic Hand, in: Proceedings of the 2002 IEEE International Conference on Robotics and Automation, Washington, DC, 3374-3379, 2002.

Rakic, M.: Multifingered Robot Hand with Selfadaptability, Rob. Comput. Integr. Manuf., 3, 269-276, 1989.

Shimojima, H., Yamamoto, K., and Kawakita, K.: A Study of Grippers with Multiple Degrees of Mobility, JSME Int. J., 30, 515$522,1987$. 